\title{
World Forests from \\ Deforestation to Transition?
}

Edited by

Matti Palo and Heidi Vanhanen

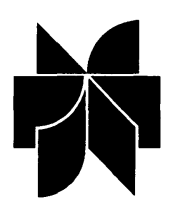

World Forests Volume II

KLUWER ACADEMIC PUBLISHERS DORDRECHT / LONDON / BOSTON 
ISBN 0-7923-6683-2

ISSN 0785-8388

Published by Kluwer Academic Publishers,

P. O. Box 17, 3300 AA Dordrecht, The Netherlands.

Sold and distributed in North, Central and South America by Kluwer Academic Publishers,

I0I Philip Drive, Norwell, MA 0206I, U.S.A.

In all other countries, sold and distributed

by Kluwer Academic Publishers,

P.O. Box 322, 3300 AH Dordrecht, The Netherlands.

Printed on acid-free paper

\begin{abstract}
All Rights Reserved
(C) 2000 Kluwer Academic Publishers

No part of the material protected by this copyright notice may be reproduced or utilized in any form or by any means, electronic or mechanical, including photocopying, recording or by any information strorage and retrieval system, without permission from the copyright owner.
\end{abstract}

Printed in Finland 


\title{
World Forests book series
}

\author{
Kluwer Academic Publishers
}

Scientific editors

Editorial Advisory Board

\author{
Matti Palo and Jussi Uusivuori \\ Finnish Forest Research Institute, \\ Helsinki, Finland
}

Mr. Philip Wardle, Chair, United Kingdom

\section{Aims and Scope}

The book series World Forests is intended for a wide range of readers as follows: national and international bodies that are concerned with forest and related policy issues, the academia - students, teachers and researchers - as well as business professionals, non-governmental organizations, the media and interested citizens worldwide. The purpose of the series is to publish research-based reviews of globally relevant issues within world forests, society and environment from an independent and non-governmental point of view.
The volumes to be published in the series will be both multidisciplined with a broad range of coverage and more focused in-depth analyses of a particular field in forest sciences. Globalization processes and comparative analyses of regions and countries are among themes to be covered. The volumes may be based on invited material or they may result from symposia, conferences, or workshops. International scientific standards will be followed. Therefore, for example, all published material will go through a peer-review process. 


\section{Preface}

This book reports one part of the findings of the research project The Forests in the South and North - Transition from Deforestation to Sustainable Forest Policies in Redressing Global Warming. The project was coordinated and partly funded by the World Institute for Development Economics Research of the United Nations University (UNU/WIDER). The Finnish Forest Research Institute (METLA) and the Institute for Applied Economic Research (IPEA/DIPES) in Brazil were among the eleven implementing institutes that contributed financially to the research. Director Eustáquio J. Reis from IPEA served as the External Project Coordinator in Rio de Janeiro while I acted as the External Project Director in Helsinki.

The policy-focused summary of the project's results was published by UNU/ WIDER in 1998 as Policy Brief No 1, Forests in Global Warming, edited by Patrick Humphreys and Matti Palo. Another part of the findings was published in June 2000 by UNU/WIDER as World Development Studies 15, Global Scenarios and Policies on Forest Transitions and Carbon Fluxes, edited by Matti Palo.

The title of this book, World Forests from Deforestation to Transition?, carries a question mark. It indicates that it may be difficult to give a final answer to this most pressing global issue. Although there are clear signs of transition in parts of the North, deforestation in the South is continuing unabated despite numerous global political efforts to control it. The problem is multisectoral and multilevel and therefore extremely complex - also very sensi- tive, due to vested national and international interests.

This book presents a set of new research findings emerging from our comprehensive research project. The book can be regarded as a follow-up to and elaboration of our previous research published in 1996 by Kluwer Academic Publishers, Sustainable Forestry Challenges for Developing Countries, edited by Matti Palo and Gerardo Mery.

To facilitate coordination, interaction and exchange of information the project organized three workshops between 1994 and 1996. The first and last workshops were held in Finland and the second one in Indonesia, in cooperation with the Center for International Forest Research (CIFOR).

The manuscript was evaluated by anonymous referees in 1997-1998, and the English language checking, editing and layout were completed in 1999-2000. During the implementation of the project the theme of this book has attained ever greater prominence on the global political agenda via the Intergovernmental Panel on Forests (IPF), the Intergovernmental Forest Forum (IFF), and the global conventions on Biodiversity and Climate Change. We hope that this volume, for its part, will offer constructive inputs to the global quest for forest transition.

Helsinki, June 2000

Matti Palo

Professor

Project Director 


\section{Acknowledgments}

Professor Mihály Simai, former Director, and Dr. Reino Hjerppe, former Principal Academic Officer of UNU/WIDER, initiated this research project. Dr. Jeffrey Sayer, Director General, and Dr. Neil Byron, Assistant Director General of CIFOR, co-financed and co-organized our second workshop in Indonesia in 1996.

Ms. Barbara Fagerman of UNU/WIDER and Ms. Margit Kuronen of METLA were in charge of financial administration and secretarial services. Dr. Ashley Selby checked the English language. Mr. Aleksi Lehtonen assisted in the finalization of the figures. Ms. Maija Heino and Ms. Essi Puranen did the layout.

We would like to express our gratitude to all the above for their excellent cooperation and professionalism. We are also thankful to the two anonymous referees who evaluated the manuscript.
Above all, we are indebted to the 14 authors who were so committed to this joint worldwide research effort. Coming from nine countries on five continents, the contributors helped to maintain the project's global, semi-global, continental and case study dimensions. The cultural and geographical diversity was our strength in tackling this most topical challenge in international forest policies.

We are very sad to announce that one of our colleagues, Dr. Beni Nasendi from Indonesia, passed away during the project. We learned to know Dr. Nasendi as a competent forest economist and a good friend, and will miss his joviality.

Helsinki, May 2000

Matti Palo Heidi Vanhanen

Editor Editor 
Note to the reader

Unit Description: the metric system is used in this publication.

- tonne $=1000 \mathrm{~kg}$

- ha $=$ hectare $\left(100 \mathrm{ha}=\right.$ one $\left.\mathrm{km}^{2}\right)$

Abbreviations:

- $\mathrm{a}=$ annum

- $\mathrm{km}=$ kilometre(s)

- $\mathrm{kg}=$ kilogram(s)

- mill. $=$ million $\left(10^{6}\right)$

- bill. = billion $\left(10^{9}\right)$

Currency: most prices and values are converted to the United States dollars (USD). Standard currency nomenclature is used for defining other currencies, when used.

Names of countries are based on FAO's classification. 


\section{Contents}

\section{PART I Global Overview and Policies}

Global Prospects on Deforestation and Forest Transition ............................. 3

by Matti Palo

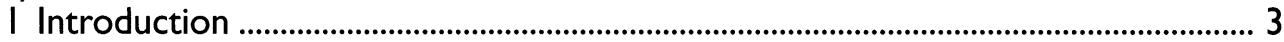

I.I World Forests Context ....................................................................................... 3

I.2 Purpose of This Volume ………………………………………………………... 5

2 Overview of the Book ........................................................................................... 6

2.I Forest Transition Prospects ……………………………………………………... 6

2.2 Causes of Pantropical Deforestation ..................................................................... 8

2.3 Deforestation in Brazil and Indonesia .................................................................. 9

2.4 Deforestation in Africa ........................................................................................ 10

3 Policy Proposals ............................................................................................................. II

3.I Deforestation Control ............................................................................................ I I

3.2 Towards Forest Transition .................................................................................. I5

\section{PART II Forest Transition}

South-North Challenges in Global Forestry ................................................... 25

by Alexander Mather

I A Time of Transition? ................................................................................................ 25

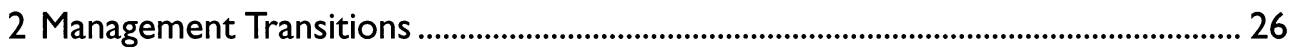

3 Forest-Area Transitions ...................................................................................... 26

4 A Changing Forest Paradigm? ................................................................................... 27

5 Global Integration:A Global Forest-Resource System ............................................... 28

5.I Internationalization of Production ..................................................................... 30

5.2 Internationalizing Trends: Supply ...................................................................... 31

5.3 Internationalizing Trends: Demand ........................................................................ 33

5.4 A Strengthening Trend? .......................................................................................... 33

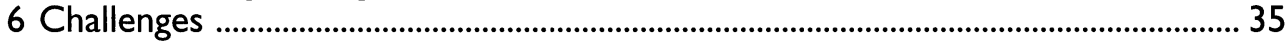

Deforestation and Forest Transition:Theory and Evidence in China .... 41 by Yaoqi Zhang

I Introduction ............................................................................................................ 4 |

2 Framework of Deforestation and Forest Transition ................................................ 42

2.I Land Use for Agriculture versus Forestry .......................................................... 42

2.2 Management of Forest Land ................................................................................. 43

2.3 Causes for Shifts in Land Use and Management .............................................. 47

3 History of Deforestation Process .............................................................................. 49

3.I Loess Plateau and Guangzhong Region ............................................................ 49

3.2 North China ................................................................................................... 50

3.3 South and Southeast China ……………………………………………………... 51

3.4 Southwest and Northeast China .......................................................................52

3.5 General Pattern of Deforestation .......................................................................... 53

4 From Deforestation to Forest Transition ................................................................5 54

5 Prospects for the Future ........................................................................................... 60

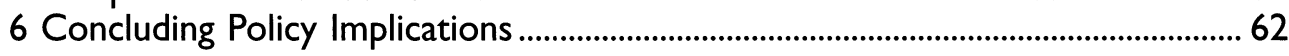




\section{From Deforestation to Reforestation}

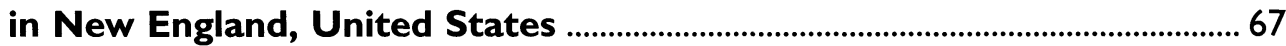

By Alexander S.P. Pfaff

I Introduction

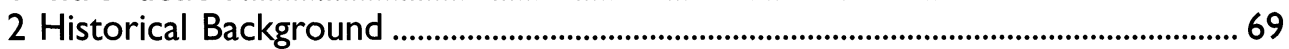

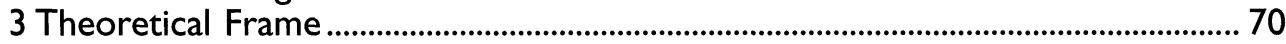

3.I Optimal Land Use, Comparative Advantage and Reforestation ................... 70

3.2 Timber: Extractive Output, Productive Land Use, and Scarce Resources 7I

3.3 Features of Manufacturing and Locational Decisions ....................................... 72

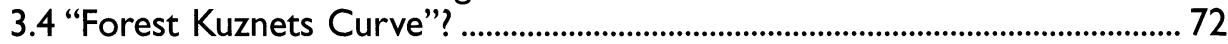

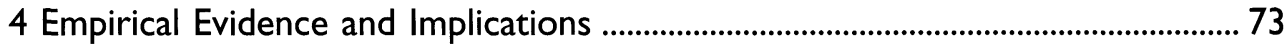

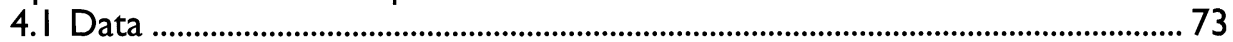

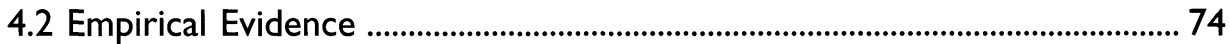

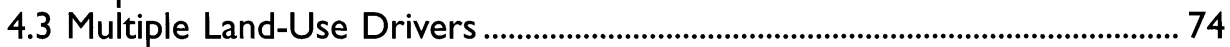

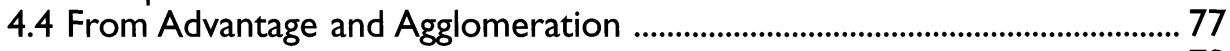

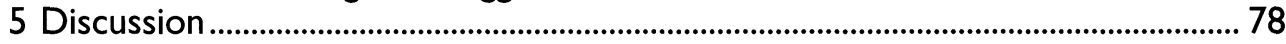

\section{Forest Plantations in the South:}

Environmental-Economic Evaluation .............................................................. 83

by Anssi Niskanen

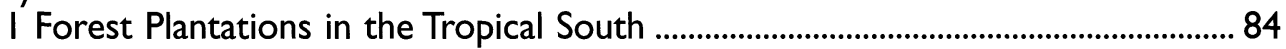

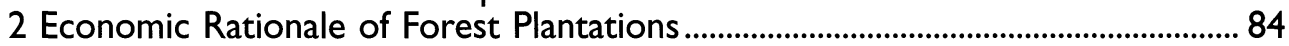

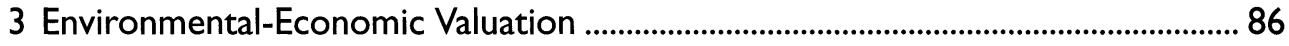

4 Case study: Acacia Plantations in the Philippines ........................................................... 88

4.I Financial and Economic Profitability Analyses ................................................ 88

4.2 Environmental-Economic Profitability ................................................................. 90

4.3 Sensitivity Analyses ...................................................................................................... 93

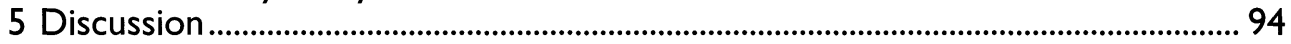

PART III Underlying Causes of Deforestation

Modeling Causes of Deforestation with 477 Subnational Units ............ 101

by Matti Palo, Erkki Lehto and Jussi Uusivuori

I Introduction

2 Model and Data ....................................................................................................... 104

2.I. Model Specification ............................................................................................ 104

2.2 Empirical Data ................................................................................................. 106

2.3 Variables .................................................................................................... 107

3 Results of Basic Modeling ............................................................................................. I II

3.I Description of Models ........................................................................................... III

3.2 Population Effects ............................................................................................. 113

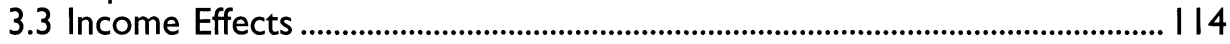

3.4 Reliability of Available Data ............................................................................. II 15

3.5 Ecological Zones............................................................................................ I I

3.6 Continental and Scaling Variables ...................................................................... II 15

4 Results of Basic Modeling Extensions ........................................................................ 116

4.I Ranking of Causal Factors ................................................................................. 116

4.2 Change Variable Modeling ................................................................................ I 18

5 Discussion ............................................................................................................ 119

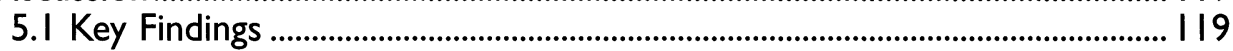

5.2 Validity and Reliability of the Results ................................................................ 120

5.3 Comparison with Earlier Findings .................................................................... 122 


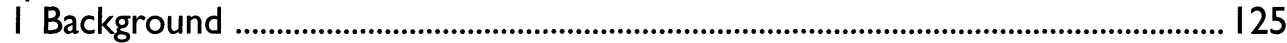

2 Modeling Deforestation ............................................................................................ 126

2.I Problem Formulation ....................................................................................... 126

2.2 The FORIS database................................................................................. $/ 27$

3 Modeling Approach in FRAI990 ............................................................................. 130

3.I Development History ........................................................................................ $|3|$

3.2 Population .................................................................................................... 132

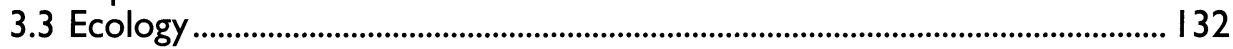

3.4 Framework for the Analysis ................................................................................. 133

4 Data and Methods ……………………………………...................................... 133

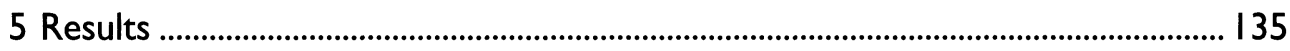

6 Discussion .......................................................................................................... 136

7 Conclusions .............................................................................................................. 138

Causes of Brazilian Amazon Deforestation ........................................................ 143

by Eustáquio J. Reis and Fernando A. Blanco

I Introduction ............................................................................................................. $\mid 43$

2 The Modernization of Agriculture ............................................................................ 144

2.I Fiscal and Credit Policies in Agriculture .......................................................... I 146

2.2 Agricultural Research .................................................................................. 147

2.3 Quantifying the Impact of Policies on Demand for Land ............................. I 147

3 Regional Development Policies ........................................................................................ 149

3.I Accessibility and Roads ....................................................................................... 149

3.2 Fiscal and Credit Incentives ........................................................................... 150

3.3 Colonization Programs ………………………………………………………... 151

3.4 Growth Poles ..................................................................................................... 152

3.5 The Rise of Environmental Concern ............................................................ 153

3.6 Quantifying the Effects of Regional Policies .................................................. 154

4 The Sources and Actors of Deforestation ............................................................ I55

4.I Agropastoral Activities .................................................................................... I 155

4.2 Squatters ...................................................................................................... 157

4.3 Cattle Ranches .............................................................................................. 159

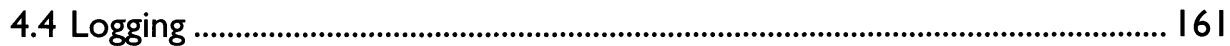

4.5 Infrastructure Construction ........................................................................ 162

5 Conclusions .............................................................................................................. 163

Deforestation and Forest Policies in Indonesia ............................................ 167

by B.D. Nasendi

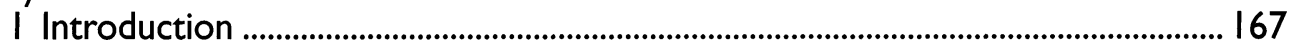

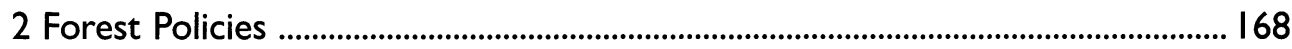

2.I Forestry Legislation ....................................................................................... 168

2.2 Forestry in National Development ............................................................... 170

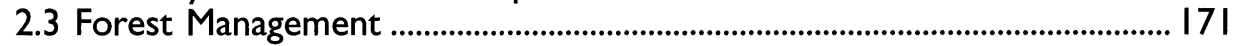

3 Dimensions of Deforestation .................................................................................... 173

4 Forestry Sector Modeling ...................................................................................... 176

4.I The Deforestation Model ................................................................................ 176

4.2 Future Scenarios .......................................................................................................... 178

5 Discussion ............................................................................................................. 180 
Deforestation and Agricultural Expansion

in Mhonda area, Tanzania

by Gerald C. Monela and Birger Solberg

I Introduction

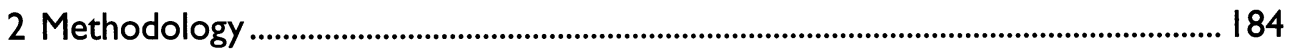

2.I Study Site and Data Collection ....................................................................... 184

2.2 Model and Main Assumptions .......................................................................... 185

3 Base Model Results Compared to Real Life Behaviour ........................................... 186

4 Results of Sensitivity Model Analysis ...................................................................... 188

4.1 Changes of Population Growth ....................................................................... 188

4.2 Impact of Changes of Working Capital Availability .......................................... 189

4.3 Impact of Agricultural Crop Price Changes ...................................................... 190

4.4 Impact of Increased Fertilizer Prices ................................................................. 190

4.5 Impact of Changes in Risk Aversion ................................................................ 191

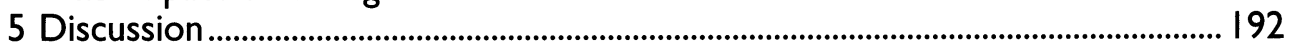

Socio-Cultural History of Deforestation in Africa ......................................... 195

by Ari Siiriäinen

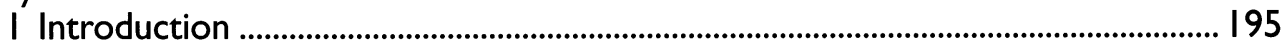

I.I Socio-History of African Deforestation ............................................................ 196

2 Population Pressure ...................................................................................................... 197

2.I Ecology and Fertility ..................................................................................... 198

2.2 The Demographic Transition Theory ................................................................ 199

2.3 What Affects Fertility Rates? ................................................................................ 199

3 History of Land Use ....................................................................................... 202

3.I Stage One: Climatic Change ............................................................................... 203

3.2 Stage Two: Incipient Cultivation ............................................................................ 203

3.3 Stage Three:Towards Intensive Resource Utilization .....................................204

3.4 Stage Four: Mixed Farming and Increasing Population ...................................205

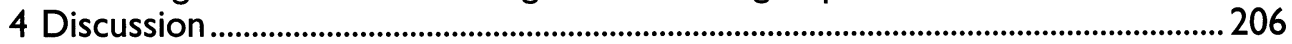

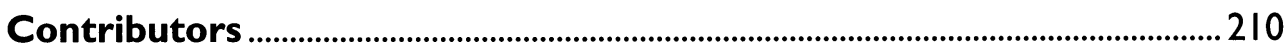

Index 